\title{
Quality assurance of non-coplanar, volumetric-modulated arc therapy employing a C-arm linear accelerator, featuring continuous patient couch rotation
}

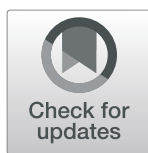

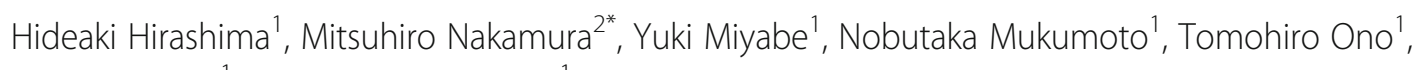
Hiraku Iramina ${ }^{1}$ and Takashi Mizowaki ${ }^{1}$

\begin{abstract}
Purpose: To perform quality assurance of non-coplanar, volumetric-modulated arc therapy featuring continuous couch rotation (CCR-VMAT) using a C-arm linear accelerator.

Methods: We planned and delivered CCR-VMAT using the TrueBeam Developer Mode. Treatment plans were created for both a C-shaped phantom and five prostate cancer patients using seven CCR trajectories that lacked collisions; we used RayStation software (ver. 4.7) to this end. Subsequently, verification plans were generated. The mean absolute error (MAE) between the center of an MV-imaged steel ball and the radiation field was calculated using the Winston-Lutz test. The MAEs between planned and actual irradiation values were also calculated from trajectory logs. In addition, correlation coefficients ( $r$ values) among the MAEs of gantry angle, couch angle, and multi-leaf collimator (MLC) position, and mechanical parameters including gantry speed, couch speed, MLC speed, and beam output, were estimated. The dosimetric accuracies of planned and measured values were also assessed using ArCCHECK.

Results: The MAEs \pm 2 standard deviations as revealed by the Winston-Lutz test for all trajectories were $0.3 \pm 0.3$ $\mathrm{mm}$ in two dimensions. The MAEs of the gantry, couch, and MLC positions calculated from all trajectory logs were within $0.04^{\circ}, 0.08^{\circ}$, and $0.02 \mathrm{~mm}$, respectively. Deviations in the couch angle $(r=0.98, p<0.05)$ and MLC position $(r=0.86, p<0.05)$ increased significantly with speed. The MAE of the beam output error was less than $0.01 \mathrm{MU}$. The mean gamma passing rate \pm 2 SD (range) of the $3 \% / 3 \mathrm{~mm}, 3 \% / 1 \mathrm{~mm}$, and $5 \% / 1 \mathrm{~mm}$ was $98.1 \pm 1.9 \%$ (95.7-99.6\%), $87.2 \pm 2.8 \%(80.2-96.7 \%)$, and $96.3 \pm 2.8 \%$ (93.9-99.6\%), respectively.

Conclusions: CCR-VMAT delivered via the TrueBeam Developer Mode was associated with high-level geometric and mechanical accuracy, thus affording to high dosimetric accuracy. The CCR-VMAT performance was stable regardless of the trajectory chosen.
\end{abstract}

Keywords: Non-coplanar VMAT, Continuous couch rotation, Developer mode, Quality assurance

\footnotetext{
* Correspondence: m_nkmr@kuhp.kyoto-u.ac.jp

${ }^{2}$ Division of Medical Physics, Department of Information Technology and Medical Engineering, Human Health Sciences, Graduate School of Medicine, Kyoto University, 53 Kawahara-cho, Shogoin, Sakyo-ku, Kyoto 606-8507, Japan Full list of author information is available at the end of the article
}

(c) The Author(s). 2019 Open Access This article is distributed under the terms of the Creative Commons Attribution 4.0 International License (http://creativecommons.org/licenses/by/4.0/), which permits unrestricted use, distribution, and reproduction in any medium, provided you give appropriate credit to the original author(s) and the source, provide a link to the Creative Commons license, and indicate if changes were made. The Creative Commons Public Domain Dedication waiver (http://creativecommons.org/publicdomain/zero/1.0/) applies to the data made available in this article, unless otherwise stated. 


\section{Introduction}

Today, $4 \pi$ radiotherapy is recognized as a useful therapeutic approach ensuring target-dose conformity while sparing doses to organs-at-risk (OARs). The dosimetric advantages of such therapy compared with coplanar intensity-modulated radiotherapy (IMRT) and volumetricmodulated arc therapy (VMAT) have been demonstrated in planning studies for many diseased sites including the brain [1, 2], head and neck [3, 4], liver [5], lung [6], breast [7] and prostate [8]. Recently, $4 \pi$ radiotherapy including $4 \pi$ static beam radiotherapy and $4 \pi$ arc beam radiotherapy has been clinically implemented using mono-isocentric beams. In a Phase 1 trial, Yu et al. showed that $4 \pi$ static beam radiotherapy was feasible and safe and associated with dosimetric benefits and high-level delivery efficiency when used to treat high-grade glioma [9]. One form of $4 \pi$ arc beam radiotherapy, O-ring system-specific non-coplanar VMAT, termed Dynamic WaveArc (DWA), has been clinically implemented in the Vero4DRT system (Mitsubishi Heavy Industries, Ltd., Hiroshima, Japan; and BrainLAB AG, Munich, Germany) [10, 11]. DWA is a continuous, non-coplanar beam delivery technique featuring simultaneous gantry and O-ring movement in the absence of couch rotation. High dose conformity and high-level delivery accuracy have been reported by several investigators [12-14].

However, $4 \pi$ arc beam radiotherapy using a typical C-arm linear accelerator, which has been termed non-coplanar VMAT featuring continuous patient couch rotation (CCR-VMAT) has been investigated in research settings [15-19]. Fahimian et al. and Liang et al. performed trajectory-modulated arc therapy featuring a continuously rotating couch by employing the TrueBeam Developer Mode (Varian Medical Systems, Palo Alto, CA, USA) [15, 16]. While significant dose-sparing of OARs was apparent, the OARs were not considered during optimization, and no gantry rotation occurred during beam delivery $[15,16]$. Optimized, continuous, non-coplanar arc trajectory methods have been developed by several research groups using the TrueBeam Developer Mode [17-19]. MacDonald et al. reported that the dose distributions associated with the developed trajectories (calculated using a dedicated treatment planning system [TPS]) did not consider a VMAT scenario featuring simultaneous gantry and couch rotation. In other words, those authors divided the optimized trajectory into sub-arcs and created deliverable plans by smoothing trajectories into multiple non-coplanar sub-arcs without dynamic couch rotation [17]. Wilson et al. developed an in-house software to create arbitrary trajectories and optimized the dose rate and multi-leaf collimator (MLC) leaf sequence [18]. Recently, Fix et al. developed a CCR-VMAT algorithm, including dynamic gantry, couch, collimator rotation and MLC sequence, with continuous movement while the beam is in operation [19]. Final dose distributions were calculated using the Monte
Carlo algorithm provided in the commercially available TPS $[19,20]$. However, the cited authors focused on the efficiency of VMAT during couch movement and these approaches required a dedicated, specific algorithm in the research environment of TPS. To our best knowledge, few reports have engaged in quality assurance (QA) using several non-coplanar trajectories with couch movement.

Here, we describe a procedure to generate CCR-VMAT plans, including beam setting, optimization and dose calculation, using a commercially available TPS that can create a DWA plan without a specific algorithm. We then implemented the CCR-VMAT plans via the TrueBeam Developer Mode, and assessed the geometric, mechanical and dosimetric accuracies of CCR-VMAT.

\section{Materials and methods CCR-VMAT delivery in the TrueBeam developer mode}

From 24 trajectories of DWA plans available in RayStation (ver. 4.7; RaySearch Laboratories, Stockholm, Sweden), seven non-coplanar arc trajectories lacking collisions were selected with the help of an in-house collision map prepared for use with TrueBeam when ArcCHECK (Sun Nuclear, Melbourne, FL, USA) was placed on the ExacTrac X-Ray 6D couch (BrainLAB) (Fig. 1). All trajectories featured 4-9 manipulation points, at which the direction of the couch rotation was switched. An additional movie file shows a room-view video of a representative trajectory (trajectory 1 ; see Additional file 1).

To create CCR-VMAT plans for the TrueBeam using RayStation software, we configured the TrueBeam machine model that enables beam delivery with simultaneous rotation of the gantry and couch by changing the geometric properties in the machine constraints workspace of RayStation. No new scripts or codes were required during planning. We then created the CCR-VMAT treatment plans described in the following section. Next, we created eXtensible Markup Language (XML) files for CCR-VMAT irradiation via the TrueBeam Developer Mode. The nominal upper limits of the gantry rotation speed, couch rotation speed, and MLC speed were $6.0^{\circ} / \mathrm{s}, 3.0^{\circ} / \mathrm{s}$, and $25 \mathrm{~mm} / \mathrm{s}$, respectively. In-house software was used to convert Digital Imaging and Communications in Medicine standard for Radiation Therapy (DICOM-RT) plans to XML files, which were uploaded to the TrueBeam Developer Mode. CCR-VMAT plans were then delivered.

\section{CCR-VMAT planning based on a C-shaped phantom and prostate cancer data}

To confirm the deliverability of CCR-VMAT plans, we created a plan for a C-shaped phantom [21] and for five patients with prostate cancer treated with DWA at our institution. The dose constraints for both plans were 


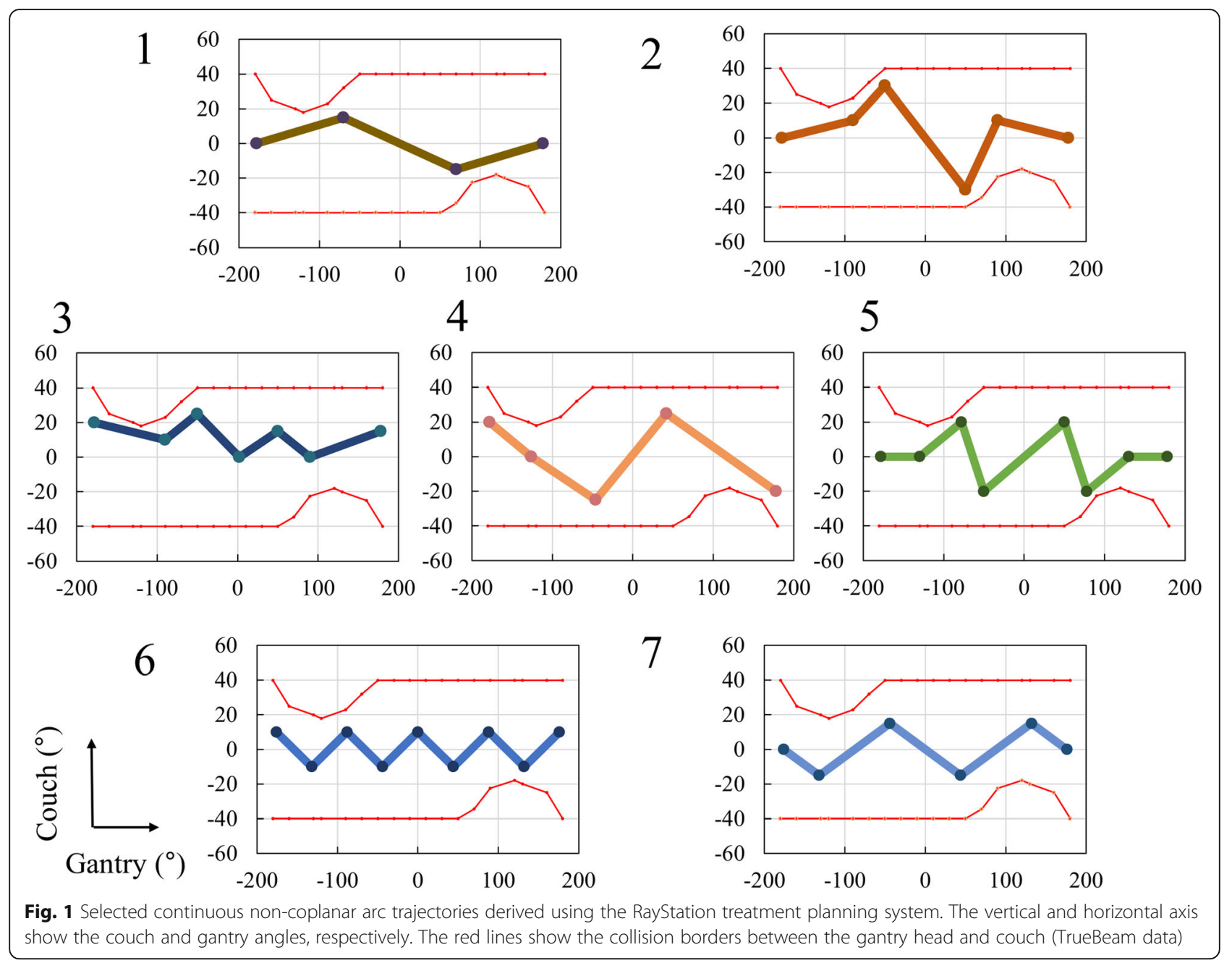

described in previous reports $[11,21]$. The prescribed doses for the $\mathrm{C}$-shaped phantom and the prostate cancer patients were $50 \mathrm{~Gy}$ in 25 fractions and $76 \mathrm{~Gy}$ in 38 fractions, respectively. All CCR-VMAT plans were created with dose calculation grid of 2-mm, using a single mono-isocentric arc. Subsequently, verification plans were generated to conduct QA.

\section{QA of CCR-VMAT}

Dosimetric inaccuracies during CCR-VMAT are caused by differences between the mechanical and radiation isocenters as well as mechanical inaccuracy. In this study, we assessed the coincidence of the mechanical and radiation isocenters using the Winston-Lutz test, as well as mechanical accuracy, such as simultaneous MLC, gantry, and couch movement, and beam output, by establishing separate trajectory logs. Thereafter, dosimetric accuracy was verified using ArcCHECK.

\section{Coincidence of mechanical and radiation isocenters} during CCR-VMAT by the Winston-Lutz test

The Winston-Lutz phantom, including a steel ball with a diameter of $3 \mathrm{~mm}$, was placed on the couch in a position defined by an in-room laser. Before the WinstonLutz test was performed, no collision between the couch and the EPID was visually confirmed for all seven trajectories.

First, two $\mathrm{kV}$ images were acquired prior to couch movement using ExacTrac (BrainLAB), to assess the difference between the actual center of the steel ball and the $\mathrm{kV}$-imaged center. Initial setup errors were not corrected. Next, a 6-MV flattened X-ray beam with a field size of $10 \times 10 \mathrm{~mm}^{2}$ was delivered to obtain electronic portal imaging device (EPID) images in continuous mode while rotating both the gantry and couch, to assess the displacement between the center of the MV-imaged steel ball and the radiation field, as described in detail in the next section. The EPID source-imager distance and pixel size were $1500 \mathrm{~mm}$ and $0.22 \times 0.22 \mathrm{~mm}^{2}$ at the isocenter, 
respectively. Finally, two more kV images were acquired by ExacTrac after the couch movement, to confirm the reproducibility of the couch position after rotation.

Based on the obtained EPID images, the displacement between the center of the MV-imaged steel ball and the radiation field during CCR-VMAT was calculated using the Winston-Lutz test employing in-house MATLAB R2017b software (MathWorks, Natick, MA, USA). The center of the steel ball was detected using a templatematching method featuring cross-correlation between the MV image and the constructed template. The center of the radiation field was determined as the center of the $50 \%$ isodose level on the EPID. The mean absolute errors (MAEs) of the displacement between the center of the MV-imaged steel ball and the radiation field in the cross-plane, in-plane, and two-dimensional directions were calculated for all seven trajectories.

\section{Mechanical accuracy}

The mechanical accuracy of CCR-VMAT was evaluated based on trajectory logs [22]. These logs recorded the commanded and measured positions at 20-ms intervals in terms of gantry angle, couch angle, MLC position, and beam output (the latter in monitor units [MUs]), respectively. The MAEs between the planned and actual trajectory log data were calculated. In addition, correlation coefficients ( $r$ values) among the MAEs of gantry angle, couch angle, and MLC position, and mechanical parameters including gantry speed, couch speed, MLC speed, and beam output, were estimated.

\section{Dosimetric accuracy}

The calculated and measured dose distributions were assessed by global gamma analysis using ArcCHECK. The criteria were $\mathrm{X} \%$ of the planned maximum dose as the dose-difference criterion and Y $\mathrm{mm}$ as the distanceto-agreement criterion, with a $10 \%$ threshold. In this study, global $3 \% / 3 \mathrm{~mm}, 3 \% / 1 \mathrm{~mm}$ and $5 \% / 1 \mathrm{~mm}$ gamma were used to assess the dose distribution. The relationships between mechanical errors and passing rates were also evaluated.

\section{Results}

\section{Coincidence of mechanical and radiation isocenters during CCR-VMAT}

The initial setup errors were less than $0.1 \mathrm{~mm}$ for all coordinates. The deviations calculated (employing the Winston-Lutz method) using the laser-to-MV beam, and the central displacement of the $\mathrm{kV}$-imaged phantom for each trajectory. The number of EPID images

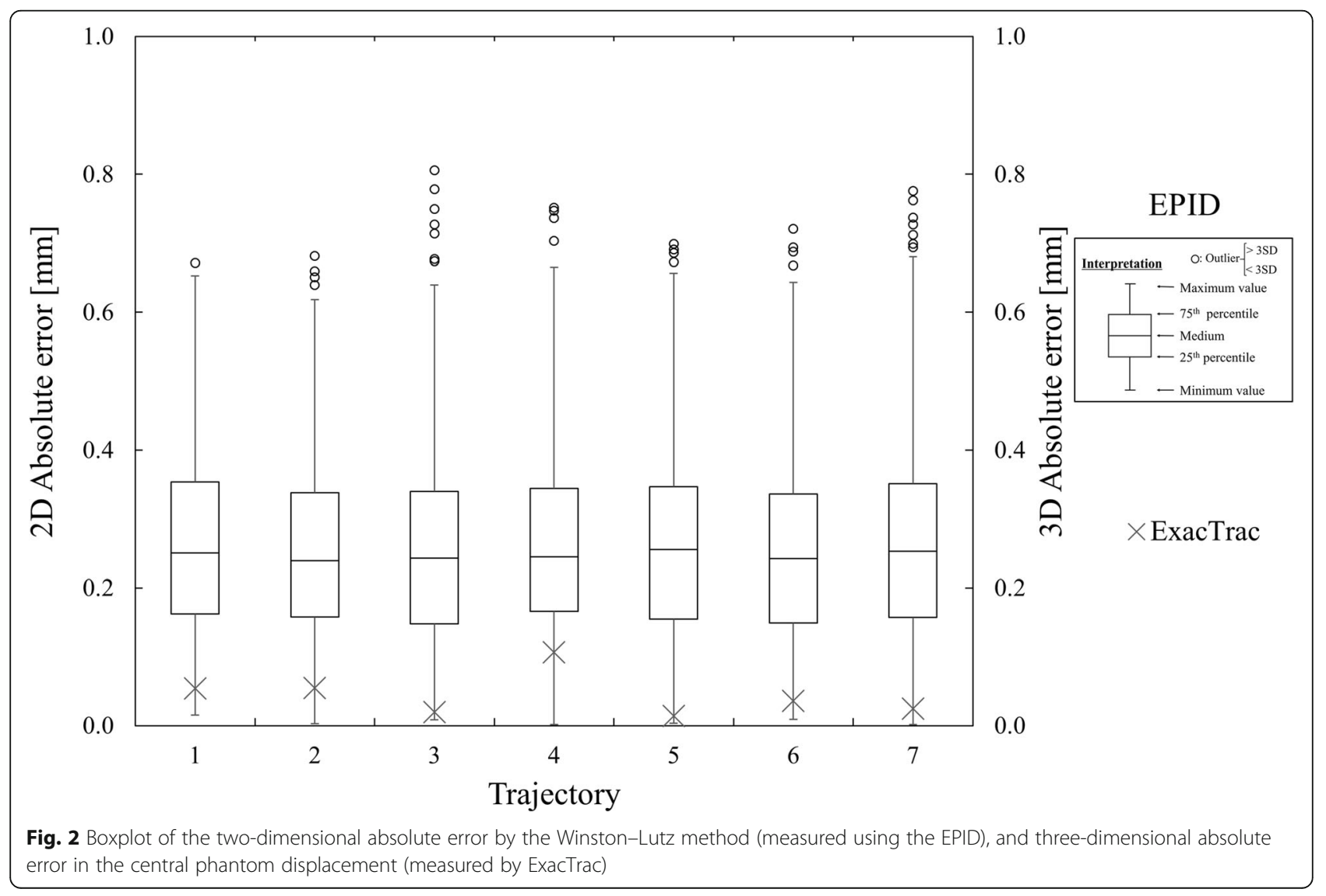




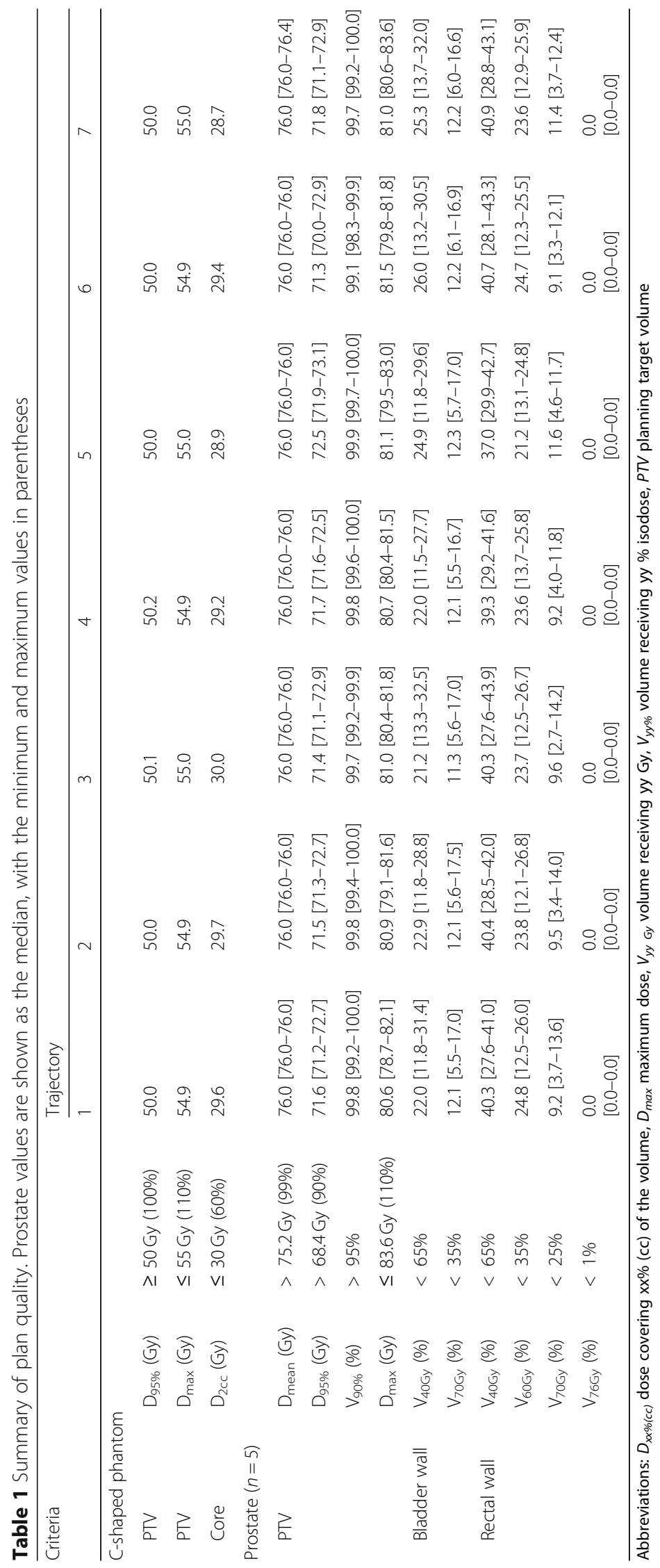


acquired during CCR-VMAT was 500 to 900 per trajectory. The MAEs \pm 2 standard deviations (SDs) (thus the maximum deviation) between the center of the MV-imaged steel ball and the radiation field were $0.2 \pm$ $0.2(0.7) \mathrm{mm}, 0.2 \pm 0.3(0.8) \mathrm{mm}$, and $0.3 \pm 0.3(0.8) \mathrm{mm}$ in the cross-plane, in-plane, and two-dimensional views, respectively. The MAEs $\pm 2 \mathrm{SD}$ (again, the maximum displacement) between the actual center of the steel ball and the $\mathrm{kV}$-imaged center before and after couch movement were $0.01 \pm 0.01(0.02) \mathrm{mm}, 0.03 \pm 0.05(0.08) \mathrm{mm}$, $0.03 \pm 0.05(0.07) \mathrm{mm}$, and $0.04 \pm 0.06(0.11) \mathrm{mm}$ in the vertical, longitudinal, lateral, and three-dimensional directions, respectively. Figure 2 shows a boxplot of the two-dimensional absolute error calculated by EPID, and the three-dimensional absolute error calculated by ExacTrac.

\section{Mechanical accuracy}

All CCR-VMAT plans met the indicated criteria (Table 1). For all CCR-VMAT plans, the MAEs of mechanical accuracies were within $0.04^{\circ}$ for the gantry position, $0.08^{\circ}$ for the couch position, $0.02 \mathrm{~mm}$ for the MLC position, and $0.01 \mathrm{MU}$ for the beam output. The mechanical accuracies of the gantry angles, couch angles, MLC positions, and beam outputs for all plans are summarized in Table 2. A representative CCR-VMAT trajectory $\log$ is shown in Fig. 3. The mean (maximum) speeds of the recorded gantry rotation, couch rotation, and MLC movement derived from the trajectory logs of all trajectories were $1.3(3.2)^{\circ} / \mathrm{s}, 3.6(6.0)^{\circ} / \mathrm{s}$, and 4.0 (17.5) $\mathrm{mm} / \mathrm{s}$, respectively. Figure 4 shows that deviations in couch angle $(r=0.98, p<0.05)$ and MLC position $(r=$ $0.86, p<0.05)$ increased significantly with speed. However, no correlation between the deviation of any mechanical parameter and beam output was apparent.

\section{Dosimetric accuracy}

The gamma passing rates of the CCR-VMAT plans for the $\mathrm{C}$-shaped phantom and prostate cancers are shown in Table 3. In all cases, the mean gamma passing rate \pm 2
SD (range) of the $3 \% / 3 \mathrm{~mm}, 3 \% / 1 \mathrm{~mm}$, and $5 \% / 1 \mathrm{~mm}$ was $98.1 \pm 1.9 \%$ (95.7-99.6\%), $87.2 \pm 2.8 \%$ (80.2-96.7\%), and $96.3 \pm 2.8 \%$ (93.9-99.6\%), respectively. Figure 5 shows the gamma maps and dose difference profiles of the representative prostate case with trajectory 1 . We found no correlation between the MAE of any mechanical parameter and any gamma passing rate.

\section{Discussion}

We generated CCR-VMAT plans without using a specific algorithm and performed QA for seven continuous, non-coplanar arc trajectories that lacked collisions, using the TrueBeam Developer Mode. The mechanical errors including those of gantry angle, couch angle, MLC position, and beam output during CCR-VMAT were comparable with those of previous reports [10, 18, 22, 23]; the dosimetric accuracies met the criteria of the American Association of Physicists in Medicine Task Group 119 [24].

Wilson et al. used an EPID to reveal the geometric inaccuracies caused by CCR. They determined that the Winston-Lutz differences between the static and couch rotation conditions were $0.2 \pm 0.1 \mathrm{~mm}$ and $-0.1 \pm 0.3$ $\mathrm{mm}$ in the cross- and in-plane directions, respectively [22]. The laser-to-MV beam errors in synchronized gantry-couch rotation were $0.2 \pm 0.2 \mathrm{~mm}$ and $0.2 \pm 0.3$ $\mathrm{mm}$ in the cross- and in-plane directions, respectively. Our experiments were more complicated than those of an earlier study since the direction of couch rotation switched 4-9 times during gantry rotation [22]; however, the deviations were comparable to those noted in that report and were stable even when continuous non-coplanar trajectories were employed. Moreover, the displacement of the laser-to- $\mathrm{kV}$ isocenter measured by ExacTrac before and after couch movement was negligible (less than $0.1 \mathrm{~mm}$ ); therefore, couch position was highly reproducible.

Wilson et al. also reported that couch angular positions in dynamic couch rotation recorded in trajectory logs were accurate to within $0.05^{\circ}$ [22]. Moreover, the

Table 2 The mechanical accuracies of gantry angle, couch angle, MLC position, and MU. The mean absolute errors \pm 2 standard deviations for the C-shaped phantom and the prostate cancers are shown

\begin{tabular}{|c|c|c|c|c|c|c|c|c|}
\hline \multirow[t]{2}{*}{ Trajectory } & \multicolumn{4}{|c|}{ C-shaped phantom } & \multicolumn{4}{|c|}{ Prostate cancers $(n=5)$} \\
\hline & Gantry $\left[^{\circ}\right]$ & Couch $\left[^{\circ}\right]$ & $\mathrm{MLC}[\mathrm{mm}]$ & Beam output [MU] & Gantry [ $\left.{ }^{\circ}\right]$ & Couch $\left[^{\circ}\right]$ & $\mathrm{MLC}[\mathrm{mm}]$ & Beam output [MU \\
\hline 1 & $0.04 \pm 0.01$ & $0.01 \pm 0.01$ & $0.03 \pm 0.04$ & $0.01 \pm 0.02$ & $0.04 \pm 0.03$ & $0.04 \pm 0.02$ & $0.02 \pm 0.03$ & $0.01 \pm 0.01$ \\
\hline 2 & $0.04 \pm 0.02$ & $0.03 \pm 0.06$ & $0.03 \pm 0.05$ & $0.01 \pm 0.02$ & $0.04 \pm 0.04$ & $0.05 \pm 0.08$ & $0.02 \pm 0.03$ & $0.01 \pm 0.02$ \\
\hline 3 & $0.04 \pm 0.02$ & $0.04 \pm 0.09$ & $0.03 \pm 0.05$ & $0.01 \pm 0.01$ & $0.04 \pm 0.02$ & $0.03 \pm 0.06$ & $0.02 \pm 0.03$ & $0.01 \pm 0.01$ \\
\hline 4 & $0.04 \pm 0.01$ & $0.03 \pm 0.03$ & $0.03 \pm 0.04$ & $0.01 \pm 0.02$ & $0.04 \pm 0.03$ & $0.07 \pm 0.05$ & $0.02 \pm 0.04$ & $0.01 \pm 0.01$ \\
\hline 5 & $0.04 \pm 0.02$ & $0.04 \pm 0.08$ & $0.03 \pm 0.05$ & $0.01 \pm 0.01$ & $0.04 \pm 0.04$ & $0.06 \pm 0.09$ & $0.02 \pm 0.03$ & $0.01 \pm 0.01$ \\
\hline 6 & $0.04 \pm 0.01$ & $0.02 \pm 0.01$ & $0.02 \pm 0.03$ & $0.01 \pm 0.02$ & $0.04 \pm 0.03$ & $0.08 \pm 0.03$ & $0.02 \pm 0.04$ & $0.01 \pm 0.01$ \\
\hline 7 & $0.04 \pm 0.02$ & $0.04 \pm 0.01$ & $0.03 \pm 0.04$ & $0.01 \pm 0.02$ & $0.04 \pm 0.03$ & $0.06 \pm 0.02$ & $0.02 \pm 0.04$ & $0.01 \pm 0.01$ \\
\hline
\end{tabular}

Abbreviations: CCR-VMAT non-coplanar, volumetric-modulated arc therapy featuring continuous couch rotation, MLC multi-leaf collimator, $M U$ monitor units 


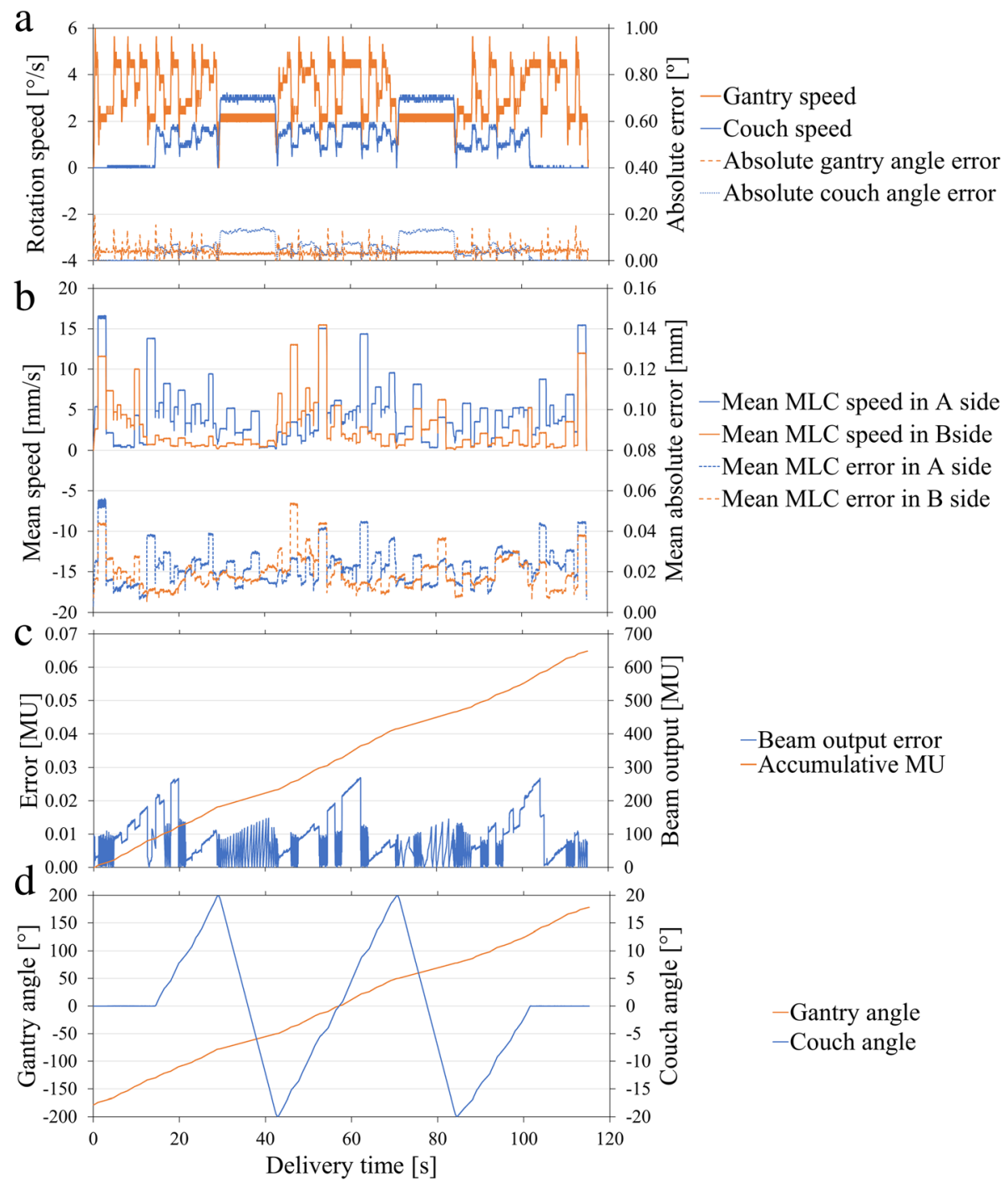

Fig. 3 The trajectory log for a representative patient treated using trajectory 5. Mechanical parameters of a the gantry and couch, $\mathbf{b}$ the MLC, $\mathbf{c}$ the beam output and, $\mathbf{d}$ the trajectory

root mean square errors of gantry and couch positions in simultaneous gantry-couch rotation attained $0.05^{\circ}$ and $0.06^{\circ}$, respectively [18]. Burghelea et al. found that the mean deviations of gantry and ring angles in DWA were $-0.03^{\circ} \pm 0.46^{\circ}$ and $0.18^{\circ} \pm 0.26^{\circ}$, respectively [10]. These results were comparable with the deviations of gantry and couch positions in CCR-VMAT recorded in our trajectory logs. Even when the MLC positions and beam outputs varied by the trajectories, the mechanical accuracy was comparable with that found in previous reports [23]. We found a high correlation between mechanical error and speed, indicating that restricting the mean or maximum mechanical speeds reduced mechanical errors, as reported previously [23]. In addition, we found no correlation between the deviation of any mechanical parameter and beam output, suggesting that the output was well controlled (thus not affected by any mechanical parameter). In terms of verification using a trajectory log, Neal et al. reported a clinically evident discrepancy between the image-based and trajectory log-based MLC positions [25]. We carefully verified this before commencing our study. We included an intentional error, which confirmed that the difference between the EPID measurement and the trajectory log was within the allowable range. Therefore, trajectory-log verification was deemed to be of adequately accurate.

In general, continuous, non-coplanar arc plans that optimize conformal dose distributions are not easily formulated, because it is difficult to avoid collisions between the gantry and couch. Existing, applicable, clinical non-coplanar arc plans are time-consuming because of the need to rotate the patient couch manually. It is well established 


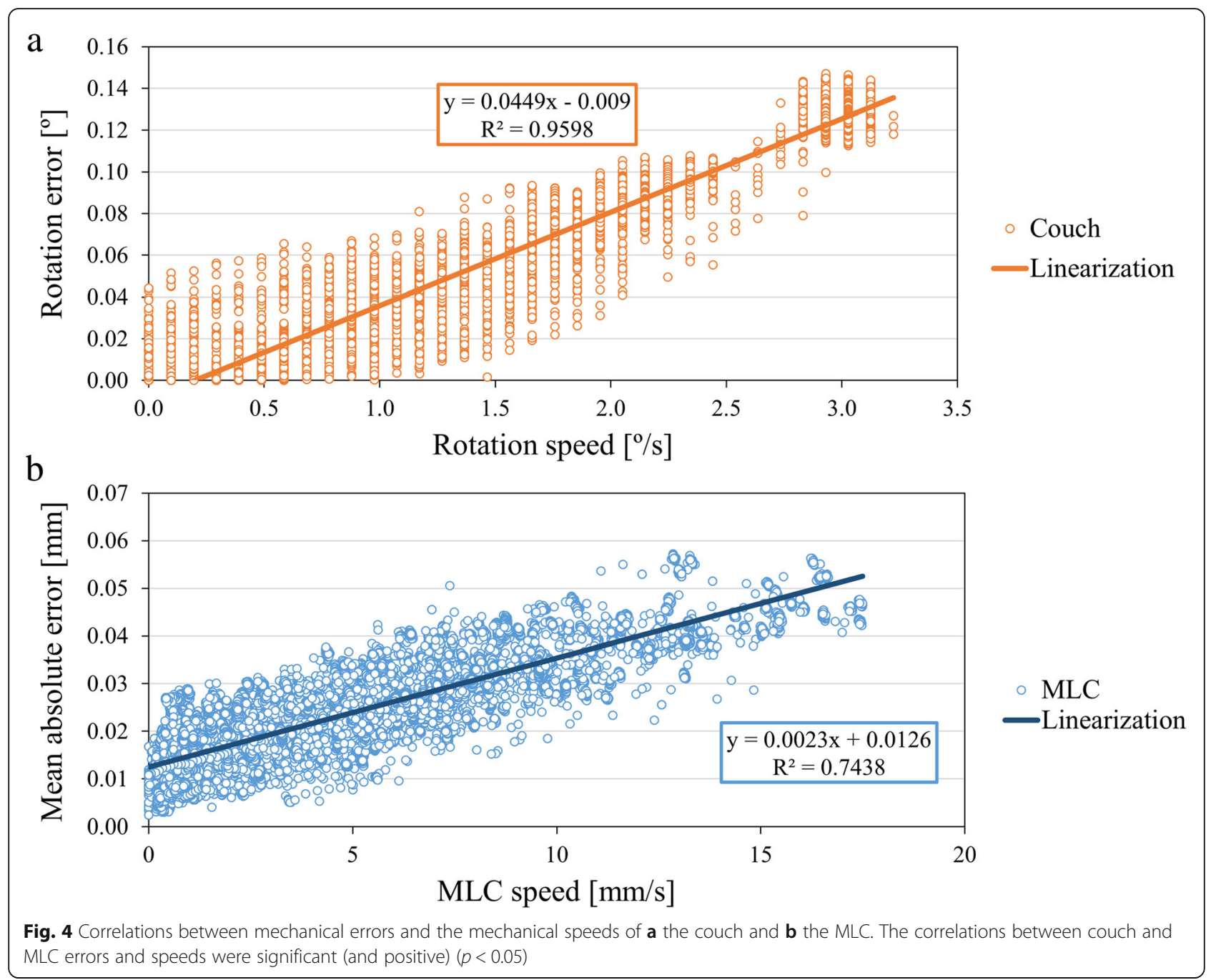

that CCR-VMAT provides dose distributions, thus enabling the concentrated administration of a sufficient dose within the target volume while minimizing the dose in the surrounding OARs, in addition to the benefit of time-saving [3, 7, 15-19]. For safe delivery of CCR-VMAT plans, dosimetric verification should be a requirement.
The average passing rates at the $3 \% / 3-\mathrm{mm}$ gamma criterion, applicable in continuous clinical non-coplanar arc delivery, were greater than $97 \%$ for several trajectories [10]. Our experimental results were comparable with the clinical results, despite the combination of TPSs and treatment machines from different vendors [26]. Therefore,

Table 3 Gamma passing rate of CCR-VMAT plans for the C-shaped phantom and prostate cancers. The prostate cancer data are means \pm 2 standard deviations

\begin{tabular}{|c|c|c|c|c|c|c|}
\hline \multirow[t]{2}{*}{ Trajectory } & \multicolumn{3}{|c|}{ C-shaped phantom } & \multicolumn{3}{|c|}{ Prostate cancers $(n=5)$} \\
\hline & $3 \% / 3$ mm [\%] & $3 \% / 1$ mm [\%] & $5 \% / 1 \mathrm{~mm}[\%]$ & $3 \% / 3$ mm [\%] & $3 \% / 1 \mathrm{~mm}[\%]$ & $5 \% / 1 \mathrm{~mm}[\%]$ \\
\hline 1 & 98.0 & 91.5 & 97.5 & $98.3 \pm 1.7$ & $86.5 \pm 2.8$ & $95.9 \pm 1.4$ \\
\hline 2 & 99.1 & 92.1 & 98.6 & $98.2 \pm 0.5$ & $84.9 \pm 0.5$ & $95.9 \pm 2.2$ \\
\hline 3 & 99.2 & 92.0 & 98.5 & $98.4 \pm 1.9$ & $85.8 \pm 1.8$ & $95.8 \pm 0.9$ \\
\hline 4 & 99.6 & 96.7 & 99.6 & $98.5 \pm 0.8$ & $88.2 \pm 4.8$ & $96.5 \pm 2.8$ \\
\hline 5 & 99.0 & 92.5 & 98.3 & $97.8 \pm 1.0$ & $86.0 \pm 3.8$ & $96.0 \pm 2.5$ \\
\hline 6 & 98.9 & 90.2 & 98.1 & $97.5 \pm 1.6$ & $85.8 \pm 3.6$ & $95.9 \pm 2.4$ \\
\hline 7 & 99.5 & 95.8 & 99.2 & $97.2 \pm 2.3$ & $87.1 \pm 5.7$ & $95.8 \pm 1.8$ \\
\hline
\end{tabular}

Abbreviation: CCR-VMAT non-coplanar, volumetric-modulated arc therapy featuring continuous couch rotation 


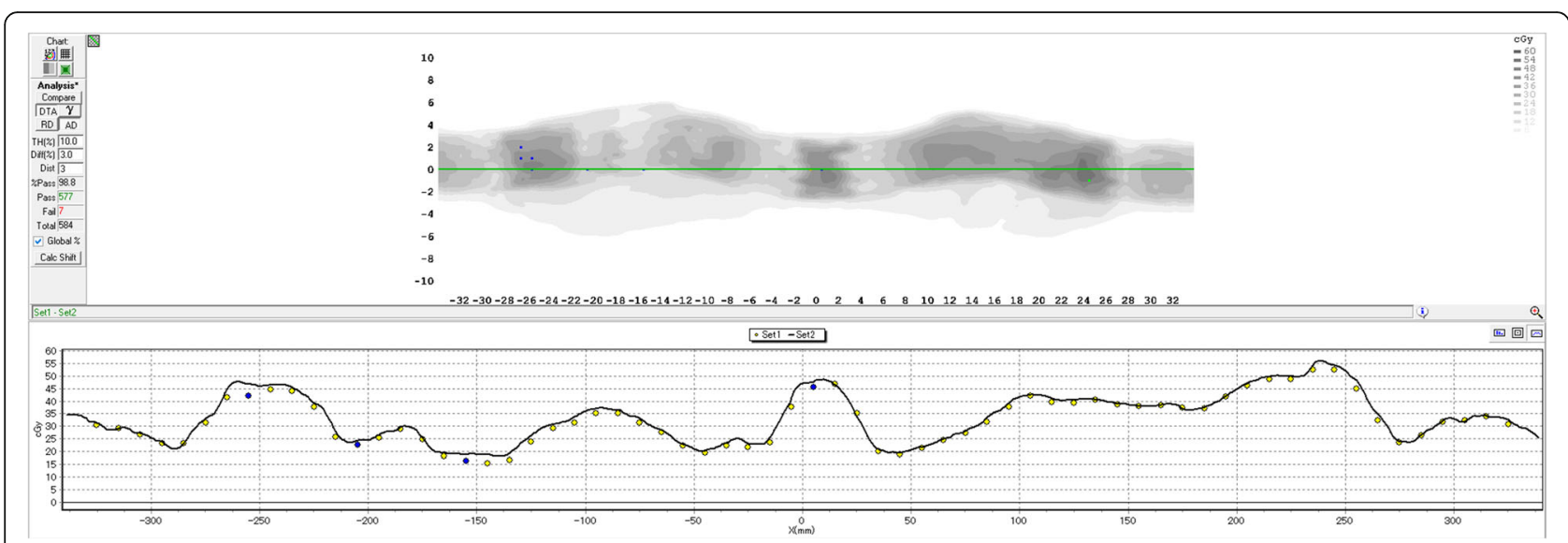

Fig. 5 An example of a gamma analysis on an ArCCHECK phantom using trajectory 1. The gamma map and dose difference profiles are shown

our approach can be expected to provide clinically acceptable and deliverable CCR-VMAT plans.

\section{Conclusion}

CCR-VMAT delivered via the TrueBeam Developer Mode was associated with high-level geometric and mechanical accuracy, which in turn afforded high dosimetric accuracy. The CCR-VMAT performance was stable regardless of the trajectory chosen.

\section{Additional files}

Additional file 1: A sample room-view video; CCR-VMAT was operated using a representative trajectory (trajectory 1). (MP4 $16236 \mathrm{~kb}$ )

Additional file 2: The dataset supporting our findings. (XLSX $3027 \mathrm{~kb}$ )

\section{Abbreviations}

CCR-VMAT: Non-coplanar VMAT featuring continuous couch rotation: DICOM-RT: Digital Imaging and Communications in Medicine standard for Radiation Therapy; DWA: Dynamic WaveArc; EPID: Electronic portal imaging device; IMRT: Intensity-modulated radiotherapy; MAEs: Mean absolute errors; MLC: Multi-leaf collimator; MUs: Monitor units; OARs: Organs at risk: PTV: Planning target volume; SD: Standard deviation; TPS: Treatment planning system; VMAT: Volumetric modulated radiation therapy; XML: eXtensible Markup Language

\section{Acknowledgments}

None.

\section{Funding}

This research was partially supported by the Practical Research for Innovative Cancer Control (grant no. JP18ck0106427h0001) of the Japan Agency for Medical Research and Development (AMED).

\section{Availability of data and materials}

Additional file 1: A sample room-view video; CCR-VMAT was operated using a representative trajectory.

Additional file 2: The dataset supporting the conclusions of this article is included in the supplementary material.

\section{Authors' contributions}

$\mathrm{HH}$ performed the planning study and statistical analysis, and drafted the manuscript. MN, YM, NM, TO, HI and TM conceived the study, participated in its design and coordination, and helped to draft the manuscript. All authors read and approved the final manuscript.

Ethics approval and consent to participate

This study followed all dictates of the Declaration of Helsinki and the Ethics Review Board of Kyoto University Hospital, and the Faculty of Medicine approved the research (approval number R1446). Written consent to participate was obtained from the patient.

\section{Consent for publication}

Written consent was obtained from the patient for publication of this report and any accompanying images.

\section{Competing interests}

The authors declare that they have no competing interests.

\section{Publisher's Note}

Springer Nature remains neutral with regard to jurisdictional claims in published maps and institutional affiliations.

\section{Author details}

'Department of Radiation Oncology and Image-applied Therapy, Graduate School of Medicine, Kyoto University, 54 Kawahara-cho, Shogoin, Sakyo-ku, Kyoto 606-8507, Japan. ${ }^{2}$ Division of Medical Physics, Department of Information Technology and Medical Engineering, Human Health Sciences, Graduate School of Medicine, Kyoto University, 53 Kawahara-cho, Shogoin, Sakyo-ku, Kyoto 606-8507, Japan.

Received: 5 November 2018 Accepted: 27 March 2019

Published online: 11 April 2019

\section{References}

1. Nguyen D, Rwigema J-CM, Yu VY, Kaprealian T, Kupelian P, Selch M, et al. Feasibility of extreme dose escalation for glioblastoma multiforme using $4 \pi$ radiotherapy. Radiat Oncol. 2014:9:1-8.

2. Yang Y, Zhang P, Happersett L, Xiong J, Yang J, Chan M, Beal K, et al. Choreographing couch and collimator in volumetric modulated arc therapy. Int J Radiat Oncol Biol Phys. 2011;80:1238-47.

3. Krayenbuehl J, Davis JB, Ciernik F. Dynamic intensity-modulated noncoplanar arc radiotherapy (INCA) for head and neck cancer. Radiat Oncol. 2006:81:1515-157.

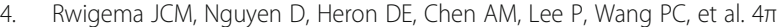
noncoplanar stereotactic body radiation therapy for head-and-neck cancer: potential to improve tumor control and late toxicity. Int J Radiat Oncol Biol Phys. 2015;91:401-9.

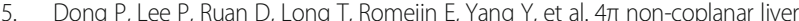
SBRT: a novel delivery technique. Int J Radiat Oncol Biol Phys. 2013;85:1360-6. 


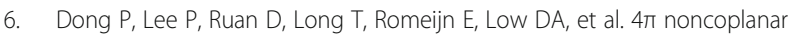
stereotactic body radiation therapy for centrally located or larger lung tumors. Int J Radiat Oncol Biol Phys. 2013;86:407-13.

7. Popescu CC, Bechham WA, Patenaude W, Olivotto Ivo A, Vlachaki MT. Simultaneous couch and gantry dynamic arc rotation (CG-Darc) in the treatment of breast cancer with accelerated partial breast irradiation (APBI): a feasibility study. J Appl Clin Med Phys. 2013;14:161-75.

8. Tran A, Zhang J, Woods K, Yu V, Nguyen D, Gustafson G, et al. Treatment planning comparison of IMPT, VMAT and $4 \pi$ radiotherapy for prostate cases. Radiat Oncol. 2017;12:1-9.

9. Yu VY, Landers A, Woods K, Nguyen D, Cao M, Du D, et al. A prospective 47 radiotherapy clinical study in recurrent high grade glioma patients. Int J Radiat Oncol Biol Phys. 2018;101:144-51.

10. Burghelea M, Verellen D, Dhont J, Hung C, Gevaert T, Van den Begin R, et al. Treating patients with dynamic wave arc: first clinical experience. Radiother Oncol. 2017;122:347-51.

11. Hirashima H, Nakamura M, Miyabe Y, Uto M, Nakamura K, Mizowaki T. Monitoring of mechanical errors and their dosimetric impact throughout the course of non-coplanar continuous volumetric-modulated arc therapy. Radiat Oncol. 2018;13:1-8.

12. Burghelea M, Verellen D, Poels $K$, Hung C, Nakamura M, Dhont J, et al. Initial characterization, dosimetric benchmark and performance validation of dynamic wave arc. Radiat Oncol. 2016;11:1-10.

13. Uto M, Mizowaki T, Ogura K, Miyabe Y, Nakamura M, Mukumoto N, et al. Volumetric modulated dynamic WaveArc therapy reduces the dose to the hippocampus in patients with pituitary adenomas and craniopharyngiomas. Pract Radiat Oncol. 2017;7:382-7.

14. Hirashima H, Nakamura M, Miyabe Y, Mukumoto N, Uto M, Nakamura K, et al. Geometric and dosimetric quality assurance using logfiles and a 3D helical diode detector for dynamic WaveArc. Phys Med. 2017:43:107-13.

15. Fahimian B, Yu V, Horst K, Xing L, Hristov D. Trajectory modulated prone breast irradiation: a LINAC-based technique combining intensity modulated delivery and motion of the couch. Radiother Oncol. 2013;109:475-81.

16. Liang J, Atwood T, Eyben RV, Fahimian B, Chin E, Horst K, et al. Trajectory modulated arc therapy: a fully dynamic delivery with synchronized couch and gantry motion significantly improves dosimetric indices correlated with poor cosmesis in accelerated partial breast irradiation. Int J Radiat Oncol Biol Phys. 2015;101:144-51.

17. MacDonald RL, Thomas CG. Dynamic trajectory-based couch motion for improvement of radiation therapy trajectories in cranial SRT. Med Phys. 2015;42:2317-25.

18. Wilson B, Otto K, Gete E. A simple and robust trajectory-based stereotactic radiosurgery treatment. Med Phys. 2017;44:240-8.

19. Fix MK, Frei D, Volken W, Terribilini D, Mueller S, Elicin O, et al. Part 1: optimization and evaluation of dynamic trajectory radiotherapy. Med Phys. 2018:45:4201-12

20. Manser $P$, Frauchiger $D$, Frei $D$, Volken $W$, Terribilini $D$, Fix MK. Dose calculation of dynamic trajectory radiotherapy using mote Carlo. Z Med Phys. 2018. https://doi.org/10.1016/j.zemedi.2018.03.002.

21. Nakamura M, Minemura T, Ishikura S, Nishio T, Narita Y, Nishimura Y. An onsite audit system for dosimetry credentialing of intensity-modulated radiotherapy in Japanese clinical oncology group (JCOG) clinical trials. Phys Med. 2016;32:987-91.

22. Wilson B, Gete E. Machine-specific quality assurance procedure for stereotactic treatments with dynamic couch rotations. Med Phys. 2017;44: 6529-37.

23. Kerns JR, Childress N, Kry SF. A multi-institution evaluation of MLC log files and performance in IMRT delivery. Radiat Oncol. 2014;9:1-10.

24. Ezzell GA, Burmeister JW, Dogan N, LoSasso TJ, Mechalakos JG, Mihailidis D, et al. IMRT commissioning: multiple institution planning and dosimetry comparisons, a report from AAPM task group 119. Med Phys. 2009:36:5359-73.

25. Neal B, Ahmed M, Kathuria K, Watkins T, Wijesooriya K, Siebers J. A clinically observed discrepancy between image-based and log-based MLC positions. Med Phys. 2016;43:2933-5.

26. Molineu A, Hernandez N, Nguyen T, Ibbott G, Followill D. Credentialing results from IMRT irradiations of an anthropomorphic head and neck phantom. Med Phys. 2013;40:022101--8.

Ready to submit your research? Choose BMC and benefit from:

- fast, convenient online submission

- thorough peer review by experienced researchers in your field

- rapid publication on acceptance

- support for research data, including large and complex data types

- gold Open Access which fosters wider collaboration and increased citations

- maximum visibility for your research: over $100 \mathrm{M}$ website views per year

At BMC, research is always in progress.

Learn more biomedcentral.com/submissions 